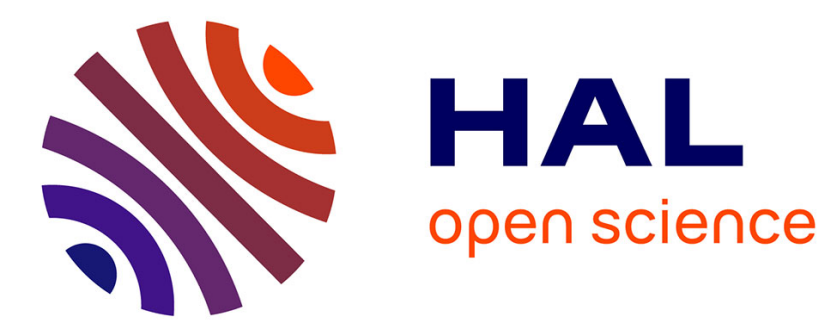

\title{
Imaging from monostatic scattered intensities
}

Jean-Baptiste Bellet, Gérard Berginc

\section{To cite this version:}

Jean-Baptiste Bellet, Gérard Berginc. Imaging from monostatic scattered intensities. Mathematical Methods in the Applied Sciences, 2014, 37 (12), pp.1772-1783. 10.1002/mma.2934 . hal-00820625

\section{HAL Id: hal-00820625 \\ https://hal.science/hal-00820625}

Submitted on 6 May 2013

HAL is a multi-disciplinary open access archive for the deposit and dissemination of scientific research documents, whether they are published or not. The documents may come from teaching and research institutions in France or abroad, or from public or private research centers.
L'archive ouverte pluridisciplinaire HAL, est destinée au dépôt et à la diffusion de documents scientifiques de niveau recherche, publiés ou non, émanant des établissements d'enseignement et de recherche français ou étrangers, des laboratoires publics ou privés. 


\title{
IMAGING FROM MONOSTATIC SCATTERED INTENSITIES
}

\author{
JEAN-BAPTISTE BELLET AND GÉRARD BERGINC
}

\begin{abstract}
This paper is about inverse-scattering from monostatic intensity-only measurements. We first formally rederive the geometrical optics approximation for a penetrable convex target. We then derive two direct imaging methods. The first one finds the point which best maps measurements over one circle onto a second one, due to amplitude decay of the scattered wave. The second one is a linearization of the problem, based on estimating the curvature of the object as a function of the measurements, due to the geometrical optics approximation. The first method aims at estimating the position of the target, whereas the second one aims at reconstructing the shape. The paper finishes with numerical tests showing the relevance and the limits of the proposed methods.
\end{abstract}

\section{INTRODUCTION}

This paper in the class of papers about the determination of the position, the shape and the electromagnetic parameters of a dielectric target using wave propagation. For such problems, signals are usually emitted by sources, and for each source, a set of receivers measures the scattered field. Several imaging algorithms have been developed for such purposes. Some of the most famous ones are the Kirchhoff migration [7], or the MUltiple Signal Classification [1]. The more recent works [2-5] are also on this subject. Both of these methods are based on the full scattered field, i.e. they need both amplitude and phase measurements in the records (in the frequency domain). However it is commonly admitted that measuring the phase may be difficult or impossible in some situations. So formulating the problem with phaseless data is an interesting problem from a practical point of view. In this paper, we will assume that the receivers record only intensities, i.e. the phase is not measured.

Let us add some comments about the mathematical effect of the loss of phase. One way to derive the Kirchhoff migration is to linearize the forward scattering problem using the Born approximation; then the linearized model leads to a convex minimization problem whose solution can be computed. Here, deleting the phase from the measurements breaks the linearity! Another point of view consists in looking at scattering data as some Fourier-type transform, and thus the inverse scattering problem is some Fourier inversion problem. Here, loosing the phase means recovering a function from its Fourier amplitude only.

Several imaging methods have been tested for intensity-only measurements. Some of them are based on phase-retrieval algorithms such as $[10,12]$. The other ones use the intensity without recovering the phase, such as $[9,14]$. Nonetheless, none of them is concerned with imaging from the scattered intensity by a penetrable target, in a monostatic configuration, i.e. with only one receiver per source, located at the source place. This is precisely the subject of this paper.

To design inversion methods, a standard approach is to study canonical models. They can indeed develop the intuition about the problem, and adapted inversion methods can easier be guessed. Eventually these methods can then be used for more general cases, either by rigorous extensions, either as heuristics. For scattering problems, a common canonical model is a high frequency asymptotics for perfectly conducting convex bodies, using a method developed in [11]. It is the so-called geometrical optics approximation. Such a model can link the monostatic radar cross-section with the curvature of the object. This is the basis of some reconstruction methods [15] formulated as the Minkowski problem: reconstructing a shape from its curvature. In the same spirit, an asymptotics is rigorously derived in [13] for a convex body with a Dirichlet, Neumann, or impedance condition, on its boundary. It is then used to analyse the inverse problem.

This leads us to study our problem with such ideas. As a canonical case, we study a high frequency asymptotics for a convex body, but with transmission conditions on its boundary. We

Date: May 6, 2013: V2.2.

2000 Mathematics Subject Classification. 78A46; 78A05.

Key words and phrases. Inverse scattering; electromagnetics; phaseless data; geometrical optics. 
will also assume that the scene is small compared to the distance of observation, and so is the distance from the object to the origin. Our derivation will be formal and will use the Kirchhoff approximation. This presentation is quite classical and is essentially derived from [7]. This leads to a formula expressing the monostatic scattered intensity as a function of two essential factors. One of them is a decay factor essentially due to the distance from the observation point to the object. The other one is the ratio of some (squared) reflection coefficient depending on the contrasts of the electromagnetic parameters, over the curvature of the object. We propose two imaging methods based on these factors. These methods have the advantage to be direct methods (to be contrasted with iterative methods of optimization).

The first one takes advantage of the intensity decay to get an original robust method wich estimates the position of the object. We have introduced in [6] an idea to reconstruct the position of an object, using monostatic scattering amplitudes over two sets of measurements. The idea is using the transport equation to map measurements of the first set onto those on the second set, using rays emanating from a small (arbitrary) disk. Then we claim that the small disk is close to the object when the mapped amplitude is close to the measured amplitude on the second set. Here, we propose the same idea, using only the order 0 (with respect to the size of the small disk) of the mapped amplitude. In fact the relevant mapping is just some intensity correction due to the intensity decay. We test the imaging function of this method under the canonical model assumption.

To find a second inversion method, we take advantage of the factor involving the curvature, by assuming that the reflection coefficient is known. We deduce a (rough) approximation of the curvature from the intensity data. This allows to formulate the problem as a differential equation whose unknown is the boundary of the object and the second member depends on the (estimated) curvature. The discrete version of this linear problem will be a linear system whose operator is a (periodic) finite differences matrix. Since the position of the object is unknown and the curvature is only roughly known, this problem is ill-posed. It is then solved using a Tikhonov-Phillips regularization [1] wich needs some a priori information, such as the position. The linear system which is finally inverted has an interesting structure: the matrix is symmetric, sparse, and circulant. To the authors' knowledge, this is an original way of formulating and solving the inverse problem of interest.

After the theoritical considerations, we test numerically the different methods. First, we test the geometrical optics approximation: we compare the scattered wave using this model with the results from the boundary element method. We test the method for different reflection coefficients and for different sizes of objects. Even if we observe results of poor quality for some parameters, we also observe that results are quite nice for a large object with a reflection coefficient close to -1 , which is close to the perfectly reflecting case (Neumann condition on the boundary of the object). So, even if the numerical results do not completely validate the forward asymptotic model, they give some meaning to the inversion methods, at least for some class of parameters.

Then we combine the imaging methods on several sets of parameters. We test more particularly the effect of the reflection coefficient, the size of the object, and the shape of the object, including non-convex objects. We observe that the first inversion method is not sensitive to the different parameters. It recovers robustly the position of the object. The second inversion method reconstructs some smooth convex curve. It is more or less close to the true object, depending essentially on the quality of the geometrical optics model.

The paper is organized as follows. We first write mathematically the imaging problem that we want to solve. Then we derive formally the geometrical optics model associated with the forward scattering model. In the next section, we interpret monostatic intensity data using this asymptotic model, and we derive the two inversion methods: the method of mapping data from one circle to another one, and the method of reconstruction from curvatures. We finish by some numerical tests about the canonical model and the inversion methods.

\section{Problem Setting}

We assume that the free space $\mathbb{R}^{2}$ is a dielectric medium whose electromagnetic parameters are the permittivity $\varepsilon_{0}>0$ and the permeability $\mu_{0}>0$. Using harmonic waves whose frequency is $\omega>0$, the wave number in free space is then $k_{0}:=\omega \sqrt{\varepsilon_{0} \mu_{0}}$, and the associated wavelength 


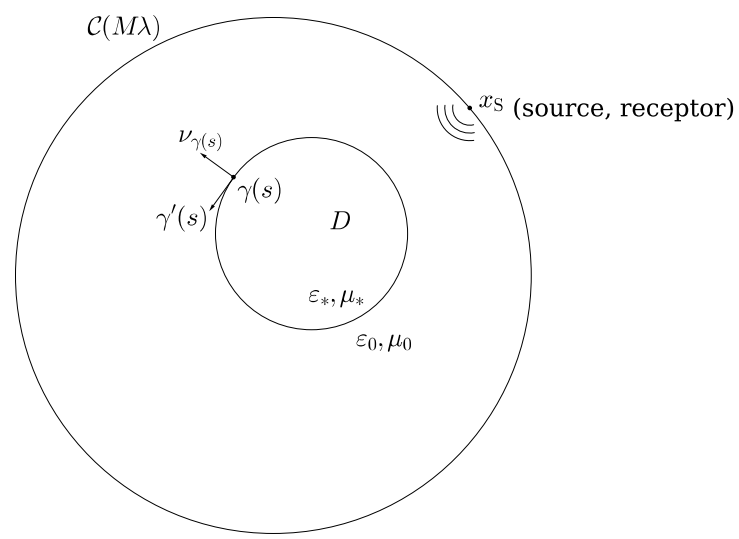

FiguRE 1. Acquisition configuration: a source at $x_{\mathrm{S}}$ on the circle $\mathcal{C}(M \lambda)$ illuminates the convex object $D$; a receptor at $x_{\mathrm{S}}$ measures the scattered intensity.

is $\lambda:=\frac{2 \pi}{k_{0}}$. A source located at some position $y$ would emit the usual outgoing Green function $G(x, y)=-\frac{\mathrm{i}}{4} \mathrm{H}_{0}^{(1)}\left(k_{0}|x-y|\right)$.

We now assume that some dielectric object $D$ is included in the medium. Its permittivity is $\varepsilon_{*}>0$ ans its permeability is $\mu_{*}>0 . D$ is supposed to be some smooth (connex) domain. Its boundary $\partial D$ can be described by a curve $\gamma(s), s$ denoting the arc length, satisfying the following properties. The unit tangent vector to $\partial D$ at $\gamma(s)$ is $\gamma^{\prime}(s)$ and the exterior unit normal vector is $\nu_{\gamma(s)}=\mathcal{R}_{-\pi / 2} \gamma^{\prime}(s)$, with $\mathcal{R}_{-\pi / 2}=\left[\begin{array}{cc}0 & 1 \\ -1 & 0\end{array}\right]$. See Figure 1. The (signed) curvature $\kappa(s)$ is such that $\gamma^{\prime \prime}=-\kappa \nu$. Except for some numerical tests, we will assume that $D$ is convex and that the curvature satisfies $\kappa \geqslant \min \kappa>0$. Also we assume that the object $D$ is extended or large, i.e. its size is a few or many wavelengths.

Let us introduce a source located at some point $x_{\mathrm{S}}$ in far field. It illuminates the object $D$ by emitting the incident field $u_{\mathrm{I}}\left(x, x_{\mathrm{S}}\right)=G\left(x, x_{\mathrm{S}}\right)$. The resulting total field can be decomposed into the form $u[D]\left(x, x_{\mathrm{S}}\right)=\left\{\begin{array}{ll}u_{\mathrm{I}}\left(x, x_{\mathrm{S}}\right)+u_{+}[D]\left(x, x_{\mathrm{S}}\right), & x \in \mathbb{R}^{2} \backslash \bar{D}, \\ u_{-}[D]\left(x, x_{\mathrm{S}}\right), & x \in D .\end{array}\right.$ The scattered field $u_{+}=$ $u_{+}[D]\left(x, x_{\mathrm{S}}\right)$ and the internal field $u_{-}=u_{-}[D]\left(x, x_{\mathrm{S}}\right)$ satisfy homogeneous Helmholtz equations, with transmission conditions on the boundary $\partial D$, and with an outgoing radiation condition:

$$
\begin{array}{r}
\left(\Delta+\omega^{2} \varepsilon_{0} \mu_{0}\right) u_{+}=0, \text { in } \mathbb{R}^{2} \backslash \bar{D}, \\
\left(\Delta+\omega^{2} \varepsilon_{*} \mu_{*}\right) u_{-}=0, \text { in } D, \\
u_{\mathrm{I}}+u_{+}=u_{-}, \text {on } \partial D, \\
\frac{1}{\mu_{0}} \partial_{\nu}\left(u_{\mathrm{I}}+u_{+}\right)=\frac{1}{\mu_{*}} \partial_{\nu} u_{-}, \text {on } \partial D, \\
+ \text { outgoing radiation condition on } u_{+} .
\end{array}
$$

We assume that a receptor located at the source position $x_{\mathrm{S}}$ measures the scattered intensity $I[D]\left(x_{\mathrm{S}}\right)=\left|u_{+}[D]\left(x_{\mathrm{S}}, x_{\mathrm{S}}\right)\right|^{2}$. In comparison, standard imaging methods such as Kirchhoff migration would use $u_{+}[D]\left(x_{\mathrm{S}}, x_{\mathrm{S}}\right)$ as input, instead of its module.

We do this experiment for a point $x_{\mathrm{S}}$ traveling along the circle $\mathcal{C}(M \lambda)$ of center 0 , and of radius is $M \lambda$. Then the data are the monostatic scattered intensities $I[D]\left(x_{\mathrm{S}}\right), x_{\mathrm{S}} \in \mathcal{C}(M \lambda)$. We will assume that the radius $M \lambda$ is large comparing with the wavelength, i.e. $M \gg 1$ is a large dimensionless number. We will also assume that the radius is large comparing with $|\gamma|$. This has two consequences: compared with an observation position $x_{S}$, the position $\hat{y}=\frac{1}{\partial D} \int_{\partial D} y \mathrm{~d} \sigma(y)$ of the object is near the origin (i.e. $|\hat{y}| \ll\left|x_{\mathrm{S}}\right|$ ), and the scene is small (i.e. $\left.|\gamma-\hat{y}| \ll\left|x_{\mathrm{S}}-\hat{y}\right|\right)$.

The aim of this paper is to study some aspects of the reconstruction of the object $D$ from the measurements $I[D](x)$. We would like tho reconstruct the shape $\gamma$ of $D$. Also, we would like to know if we can guess the electromagnetic parameters $\varepsilon_{*}, \mu_{*}$. 


\section{Asymptotic model}

3.1. Eikonal and transport equations. Let us consider a general homogeneous Helmholtz problem in a homogeneous medium :

$$
\left(\Delta+\omega^{2} \varepsilon \mu\right) u=0
$$

where $\omega$ is the frequency, $\sqrt{\varepsilon \mu}>0$ is a constant representing the slowness of the medium. In order to derive the eikonal and transport equation in high frequency regime, let us write formally the WKBJ expansion of $u$ :

$$
u(x) \sim \omega^{\beta} \mathrm{e}^{\mathrm{i} \omega \varphi(x)} \sum_{j=0}^{\infty} \frac{A_{j}(x)}{(\mathrm{i} \omega)^{j}} .
$$

This expression is some harmonic version of a progressive wave $F(t-\varphi(x))$ propagating in the direction of increasing $\varphi(x)$ (in time domain). So the phase function $\varphi(x)$ corresponds to a travel time. The constant power $\beta$ depends on some kind of initial conditions imposed on $u$, such as the source signature. The terms of the sum involve amplitude terms $A_{j}(x)$ which do not depend on $\omega$. They are smoother and smoother, since a division by $\mathrm{i} \omega$ represents an integration in time domain; thus the sharpest part, or somehow the highest frequency part, comes from the first terms of the expansion. We will use in the sequel leading order approximations for such expansions: $u(x) \sim \omega^{\beta} \mathrm{e}^{\mathrm{i} \omega \varphi(x)} A_{0}(x)$.

Insertion of the WKBJ expansion (3.2) in the Helmholtz equation (3.1) yields:

$$
\omega^{\beta} \mathrm{e}^{\mathrm{i} \omega \varphi} \sum_{j=0}^{\infty} \frac{1}{(\mathrm{i} \omega)^{j}}\left[\omega^{2}\left(\varepsilon \mu-|\nabla \varphi|^{2}\right) A_{j}+\mathrm{i} \omega\left(2 \nabla \varphi \cdot \nabla A_{j}+A_{j} \Delta \varphi\right)+\Delta A_{j}\right]=0 .
$$

Let us identify formally powers of $\omega$. The highest power $\beta+2$ yields the eikonal equation which claims that the slowness is the norm of the gradient of the phase; The power $\beta$ yields the transport equation, which links the amplitude $A_{0}$ with the phase:

$$
|\nabla \varphi|^{2}-\varepsilon \mu=0, \quad 2 \nabla \varphi \cdot \nabla A_{0}+A_{0} \Delta \varphi=0 .
$$

For our purpose, we won't need to solve neither the eikonal equation nor the transport equation. We only need to focus on what such high frequency asymptotics implies at an interface between two media.

3.2. Reflection and transmission at an interface. In this subsection, we consider an interface $\Gamma$ separating two homogeneous medium. The electromagnetic parameters are $\varepsilon_{0}, \mu_{0}$ above the interface and $\varepsilon_{*}, \mu_{*}$ below the interface. We assume that a downward incident wave $u_{\mathrm{I}}$ in the upper part generates at the boundary an upward reflected wave $u_{\mathrm{R}}$ in the upper part, and a downward transmitted wave $u_{\mathrm{T}}$ in the lower medium. Of course, $u_{\mathrm{I}}, u_{\mathrm{R}}$ satisfy a Helmholtz equation such as (3.1) with $\varepsilon \mu=\varepsilon_{0} \mu_{0}$, and $u_{\mathrm{T}}$ satisfies such an equation with $\varepsilon \mu=\varepsilon_{*} \mu_{*}$. So we can write the WKBJ expansions (3.2) of $u_{\mathrm{I}, \mathrm{R}, \mathrm{T}}$, and we apply the results of the previous subsection (we replace $\beta$, $\varphi$ and $A_{j}$ by $\beta_{\mathrm{I}, \mathrm{R}, \mathrm{T}}, \varphi_{\mathrm{I}, \mathrm{R}, \mathrm{T}}$ and $A_{j}^{\mathrm{I}, \mathrm{R}, \mathrm{T}}$ ). Furthermore, $u_{\mathrm{I}}, u_{\mathrm{R}}$ and $u_{\mathrm{T}}$ must satisfy the two following transmission conditions on the interface:

$$
u_{\mathrm{I}}+u_{\mathrm{R}}=u_{\mathrm{T}}, \quad \frac{1}{\mu_{0}} \partial_{\nu}\left(u_{\mathrm{I}}+u_{\mathrm{R}}\right)=\frac{1}{\mu_{*}} \partial_{\nu} u_{\mathrm{T}}, \quad \text { on } \Gamma .
$$

Our main goal in this subsection is to express the reflected field $u_{\mathrm{R}}$ and its normal derivative $\partial_{\nu} u_{\mathrm{R}}$ on the interface as a function of the incident field $u_{\mathrm{I}}$ and its normal derivative $\partial_{\nu} u_{\mathrm{I}}$. We obtain these results at the leading order, using the transmission conditions.

The first transmission condition in (3.4) becomes:

$$
\omega^{\beta_{\mathrm{I}}} \mathrm{e}^{\mathrm{i} \omega \varphi_{\mathrm{I}}} \sum_{j=0}^{\infty} \frac{A_{j}^{\mathrm{I}}}{(\mathrm{i} \omega)^{j}}+\omega^{\beta_{\mathrm{R}}} \mathrm{e}^{\mathrm{i} \omega \varphi_{\mathrm{R}}} \sum_{j=0}^{\infty} \frac{A_{j}^{\mathrm{R}}}{(\mathrm{i} \omega)^{j}}=\omega^{\beta_{\mathrm{T}}} \mathrm{e}^{\mathrm{i} \omega \varphi_{\mathrm{T}}} \sum_{j=0}^{\infty} \frac{A_{j}^{\mathrm{T}}}{(\mathrm{i} \omega)^{j}} \text {, on } \Gamma \text {. }
$$

In general, terms of different order in $\omega$ cannot be equal, so $\beta_{\mathrm{I}}=\beta_{\mathrm{R}}=\beta_{\mathrm{T}}$. We must also have $\varphi_{\mathrm{I}}=\varphi_{\mathrm{R}}=\varphi_{\mathrm{T}}$ on $\Gamma$ to be able to match term of like power in $\omega$. This determines the phase for the fields $u_{\mathrm{R}}$ and $u_{\mathrm{T}}$ on the interface. As phases are equal on the interface, their tangential derivatives are also equal: $\partial_{\tau} \varphi_{\mathrm{I}}=\partial_{\tau} \varphi_{\mathrm{R}}=\partial_{\tau} \varphi_{\mathrm{T}}$. By the way, the eikonal equation in (3.3) implies that $\left|\nabla \varphi_{\mathrm{I}}\right|^{2}=\left|\nabla \varphi_{\mathrm{R}}\right|^{2}=\varepsilon_{0} \mu_{0}$, and $\left|\nabla \varphi_{\mathrm{T}}\right|^{2}=\varepsilon_{*} \mu_{*}$. First, substracting the second equality to the 
first one yields: $\left(\partial_{\nu} \varphi_{\mathrm{T}}\right)^{2}=\varepsilon_{*} \mu_{*}-\varepsilon_{0} \mu_{0}+\left(\partial_{\nu} \varphi_{\mathrm{I}}\right)^{2}$. But $u_{\mathrm{I}}$ and $u_{\mathrm{T}}$ are both downward waves, so $\partial_{\nu} \varphi_{\mathrm{I}}$ and $\partial_{\nu} \varphi_{\mathrm{T}}$ have the same sign, and thus

$$
\partial_{\nu} \varphi_{\mathrm{T}}=\operatorname{sign}\left(\partial_{\nu} \varphi_{\mathrm{I}}\right) \sqrt{\varepsilon_{*} \mu_{*}-\varepsilon_{0} \mu_{0}+\left(\partial_{\nu} \varphi_{\mathrm{I}}\right)^{2}} .
$$

Secondly, as $\nabla \varphi_{\mathrm{I}}$ and $\nabla \varphi_{\mathrm{R}}$ have the same norm and the same tangential component, their normal component are equal, up to the sign. As $u_{\mathrm{I}}$ is a downward wave and $u_{\mathrm{R}}$ is an upward wave, they are opposite:

$$
\partial_{\nu} \varphi_{\mathrm{R}}=-\partial_{\nu} \varphi_{\mathrm{I}}
$$

At this step, we know the phase and its gradient, for the reflected and transmitted fields on the interface, as a function of the incident field. Let us determine now the amplitude. Since the powers $\beta$ are the same, and so are the phases $\varphi$, the WKBJ expansions inserted into the transmission conditions (3.4) yield:

$$
\begin{aligned}
& \sum_{j=0}^{\infty} \frac{A_{j}^{\mathrm{I}}}{(\mathrm{i} \omega)^{j}}+\sum_{j=0}^{\infty} \frac{A_{j}^{\mathrm{R}}}{(\mathrm{i} \omega)^{j}}=\sum_{j=0}^{\infty} \frac{A_{j}^{\mathrm{T}}}{(\mathrm{i} \omega)^{j}}, \\
& \sum_{j=0}^{\infty}\left[\frac{1}{(\mathrm{i} \omega)^{j-1}}\left(\frac{1}{\mu_{0}} A_{j}^{\mathrm{I}} \partial_{\nu} \varphi_{\mathrm{I}}+\frac{1}{\mu_{0}} A_{j}^{\mathrm{R}} \partial_{\nu} \varphi_{\mathrm{R}}-\frac{1}{\mu_{*}} A_{j}^{\mathrm{T}} \partial_{\nu} \varphi_{\mathrm{T}}\right)+\right. \\
&\left.\frac{1}{(\mathrm{i} \omega)^{j}}\left(\frac{1}{\mu_{0}} \partial_{\nu} A_{j}^{\mathrm{I}}+\frac{1}{\mu_{0}} \partial_{\nu} A_{j}^{R}-\frac{1}{\mu_{*}} \partial_{\nu} A_{j}^{\mathrm{T}}\right)\right]=0, \text { on } \Gamma .
\end{aligned}
$$

By formally identifying like power in $\mathrm{i} \omega$ : the power 0 in the first line above yields $A_{0}^{\mathrm{I}}+A_{0}^{\mathrm{R}}=A_{0}^{\mathrm{T}}$, and the power -1 in the second line yields $\frac{1}{\mu_{0}} A_{0}^{\mathrm{I}} \partial_{\nu} \varphi_{\mathrm{I}}+\frac{1}{\mu_{0}} A_{0}^{\mathrm{R}} \partial_{\nu} \varphi_{\mathrm{R}}-\frac{1}{\mu_{*}} A_{0}^{\mathrm{T}} \partial_{\nu} \varphi_{\mathrm{T}}=0$. If we introduce the Fresnel coefficients $R$ and $T$ such that: $A_{0}^{\mathrm{R}}=R A_{0}^{\mathrm{I}}$ and $A_{0}^{\mathrm{T}}=T A_{0}^{\mathrm{I}}$, and using (3.6), these two transmission conditions are given by: $1+R=T, \frac{1}{\mu_{0}}(1-R) \partial_{\nu} \varphi_{\mathrm{I}}=\frac{1}{\mu_{*}} T \partial_{\nu} \varphi_{\mathrm{T}}$, i.e. $\quad R=$ $\frac{\frac{1}{\mu_{0}} \partial_{\nu} \varphi_{\mathrm{I}}-\frac{1}{\mu_{*}} \partial_{\nu} \varphi_{\mathrm{T}}}{\frac{1}{\mu_{0}} \partial_{\nu} \varphi_{\mathrm{I}}+\frac{1}{\mu_{*}} \partial_{\nu} \varphi_{\mathrm{T}}}, T=\frac{\frac{2}{\mu_{0}} \partial_{\nu} \varphi_{\mathrm{I}}}{\frac{1}{\mu_{0}} \partial_{\nu} \varphi_{\mathrm{I}}+\frac{1}{\mu_{*}} \partial_{\nu} \varphi_{\mathrm{T}}}$. If the opposite of the incident direction $-\nabla \varphi_{\mathrm{I}}$ makes an accute angle $\theta$ with the normal direction $\nu$, we can write $\partial_{\nu} \varphi_{\mathrm{I}}=-\sqrt{\varepsilon_{0} \mu_{0}} \cos \theta<0$. Then, we get the Fresnel coefficients from (3.5); in particular, for normal incidence $(\theta=0)$, we get coefficients depending only on the permeability and the slowness contrasts:

$$
\begin{aligned}
R_{\theta}=\frac{\frac{\mu_{*}}{\mu_{0}} \cos \theta-\sqrt{\frac{\varepsilon_{*} \mu_{*}}{\varepsilon_{0} \mu_{0}}-\sin ^{2} \theta}}{\frac{\mu_{*}}{\mu_{0}} \cos \theta+\sqrt{\frac{\varepsilon_{*} \mu_{*}}{\varepsilon_{0} \mu_{0}}-\sin ^{2} \theta}}, T_{\theta}= & \frac{2 \frac{\mu_{*}}{\mu_{0}} \cos \theta}{\frac{\mu_{*}}{\mu_{0}} \cos \theta+\sqrt{\frac{\varepsilon_{*} \mu_{*}}{\varepsilon_{0} \mu_{0}}-\sin ^{2} \theta}}, \\
& \text { and for } \theta=0: R_{0}=\frac{\frac{\mu_{*}}{\mu_{0}}-\frac{\sqrt{\varepsilon_{*} \mu_{*}}}{\sqrt{\varepsilon_{0} \mu_{0}}}}{\frac{\mu_{*}}{\mu_{0}}+\frac{\sqrt{\varepsilon_{*} \mu_{*}}}{\sqrt{\varepsilon_{0} \mu_{0}}}}, T_{0}=\frac{2 \frac{\mu_{*}}{\mu_{0}}}{\frac{\mu_{*}}{\mu_{0}}+\frac{\sqrt{\varepsilon_{*} \mu_{*}}}{\sqrt{\varepsilon_{0} \mu_{0}}}} .
\end{aligned}
$$

To conclude this section, let us have a look at some leading order terms that we got on the interface $\Gamma$. For the incident wave, we have $u_{\mathrm{I}} \sim \omega^{\beta_{\mathrm{I}}} \mathrm{e}^{\mathrm{i} \omega \varphi_{\mathrm{I}}} A_{0}^{\mathrm{I}}$. The reflected wave $u_{\mathrm{R}}$ has a similar expression with $\beta_{\mathrm{R}}=\beta_{\mathrm{I}}, \varphi_{\mathrm{R}}=\varphi_{\mathrm{I}}$ and $A_{0}^{\mathrm{R}}=R A_{0}^{\mathrm{I}}$, with $R$ given by (3.7). On the other hand, at the leading order, $\partial_{\nu} u \sim \mathrm{i} \omega u \partial_{\nu} \varphi$, for $u=u_{\mathrm{R}}$ and for $u=u_{\mathrm{I}}$. Recalling (3.6), we finally get what will be a key step in the Kirchhoff approximation:

$$
u_{\mathrm{R}} \sim R u_{\mathrm{I}}, \quad \partial_{\nu} u_{\mathrm{R}} \sim-R \partial_{\nu} u_{\mathrm{I}}
$$

3.3. Kirchhoff approximation. Let us come back to the original problem defined by (2.1), (2.2), (2.3), (2.4), (2.5). The scattered field $u_{+}$satisfies the Helmholtz equation (2.1) with the radiation condition (2.5). So it has the following Green representation [1]:

$$
u_{+}(x)=\int_{\partial D}\left(G(x, y) \partial_{\nu_{y}} u_{+}(y)-u_{+}(y) \partial_{\nu_{y}} G(x, y)\right) \mathrm{d} \sigma(y) .
$$

The idea of Kirchhoff approximation is to consider only the contributions from primary reflections on the visible part $\Gamma=\left\{y \in \partial D:\left(y-x_{\mathrm{S}}\right) \cdot \nu_{y}<0\right\}$, and to approximate them at the leading order. 
The well known leading order term of the Green function is thus of interest:

$$
\begin{aligned}
G(x, y) \sim \omega^{\beta} \mathrm{e}^{\mathrm{i} \omega \varphi(x, y)} A_{0}(x, y), \text { with } \beta=-0.5, \varphi(x, y)=\sqrt{\varepsilon_{0} \mu_{0}}|x-y|, \\
A_{0}(x, y)=\frac{-\mathrm{e}^{\mathrm{i} \pi / 4}}{\sqrt{8 \pi \sqrt{\varepsilon_{0} \mu_{0}}}} \frac{1}{\sqrt{|x-y|}} .
\end{aligned}
$$

This result gives the leading order term for the incident wave $u_{I}\left(x, x_{\mathrm{S}}\right)=G\left(x, x_{\mathrm{S}}\right)$ and the gradient of its phase: $\nabla \varphi\left(x, x_{\mathrm{S}}\right)=\sqrt{\varepsilon_{0} \mu_{0}} \frac{x-x_{\mathrm{S}}}{\left|x-x_{\mathrm{S}}\right|}$. On a visible point $y \in \Gamma$, we approximate the scattered field $u_{+}(y)$ by the reflected field $u_{\mathrm{R}}$ resulting from the interaction of the incident field $u_{\mathrm{I}}$ with the interface $\Gamma$. Since the incident wave is downgoing: $\partial_{\nu_{y}} \varphi=\sqrt{\varepsilon_{0} \mu_{0}} \frac{y-x_{\mathrm{S}}}{\left|y-x_{\mathrm{S}}\right|} \cdot \nu_{y}<0$, then following the approximation (3.8), we get:

$$
u_{+} \sim R u_{\mathrm{I}}, \quad \partial_{\nu} u_{+} \sim-R \partial_{\nu} u_{\mathrm{I}}, \text { on } \Gamma .
$$

By the way, for a point $y$ in the shadow part $\left\{y \in \partial D:\left(y-x_{\mathrm{S}}\right) \cdot \nu_{y}>0\right\}$, we assume that the scattered field and its normal derivative are very small; so we remove these contributions from the integral (3.9) which becomes an integral over the lit part $\Gamma$. Finally, inserting the approximation (3.11) in the Green representation (3.9) yields:

$$
\begin{aligned}
u_{+}(x)=-\int_{\Gamma} R\left(G(x, y) \partial_{\nu_{y}} u_{\mathrm{I}}\left(y, x_{\mathrm{S}}\right)+u_{\mathrm{I}}\left(y, x_{\mathrm{S}}\right) \partial_{\nu_{y}} G(x, y)\right) & \mathrm{d} \sigma(y) \\
= & -\int_{\Gamma} R \partial_{\nu_{y}}\left(G(x, y) u_{\mathrm{I}}\left(y, x_{\mathrm{S}}\right)\right) \mathrm{d} \sigma(y) .
\end{aligned}
$$

The leading order terms now yield:

$$
u_{+}(x)=-\mathrm{i} \int_{\Gamma} R \partial_{\nu_{y}}\left(\varphi\left(y, x_{S}\right)+\varphi(x, y)\right) A_{0}\left(y, x_{\mathrm{S}}\right) A_{0}(x, y) \mathrm{e}^{\mathrm{i} \omega\left(\varphi\left(y, x_{S}\right)+\varphi(x, y)\right)} \mathrm{d} \sigma(y) .
$$

In the monostatic case, $x=x_{\mathrm{S}}$, and so we get with the leading order of the Green function (3.10):

$$
\begin{aligned}
u_{+}(x)=\int_{\Gamma} f(s) \mathrm{e}^{\mathrm{i} \Lambda \Phi(s)} \mathrm{d} s, \text { with } f(s)=\frac{1}{4 \pi} R(\gamma(s)) \frac{\gamma(s)-x}{|\gamma(s)-x|} \cdot \nu(\gamma(s)) \frac{1}{|\gamma(s)-x|}, \\
\Phi(s)=\left|\frac{\gamma(s)}{\rho}-\frac{x}{\rho}\right|, \Lambda=2 \omega \rho \sqrt{\varepsilon_{0} \mu_{0}} .
\end{aligned}
$$

We have used the parametrization $\gamma$ to write the integral; the parameter $\rho=\frac{1}{\partial D \mid} \int_{\partial D}|y-\hat{y}| \mathrm{d} \sigma(y)$ is the average distance of the points of the boundary $\partial D$ to the location of the object. The function $\Phi$ and the parameter $\Lambda$ are dimensionless. We now perform a stationary phase analysis wich reveals that in fact there is only one visible point which contributes to this integral. If $\Lambda$ is large, the stationary phase formula yields [8]:

$$
u_{+}(x) \sim \sqrt{\frac{2 \pi}{|\Lambda|\left|\Phi^{\prime \prime}\left(s_{0}\right)\right|}} f\left(s_{0}\right) \mathrm{e}^{\mathrm{i} \Lambda \Phi\left(s_{0}\right)+\mathrm{i} \pi / 4 \operatorname{sign}(\Lambda) \operatorname{sign}\left(\Phi^{\prime \prime}\left(s_{0}\right)\right)},
$$

where $s_{0}$ is the stationary point of $\Phi: \Phi^{\prime}\left(s_{0}\right)=\frac{\gamma\left(s_{0}\right)-x}{\left|\gamma\left(s_{0}\right)-x\right|} \cdot \frac{1}{\rho} \gamma^{\prime}\left(s_{0}\right)=0$; the specular point $y_{0}=\gamma\left(s_{0}\right)$ is the visible point on $\partial D$ such that the incidence is normal on $y_{0}: \frac{y_{0}-x}{\left|y_{0}-x\right|}=-\nu_{y_{0}}$. So $f\left(s_{0}\right)=$ $\frac{R_{0}}{4 \pi} \frac{-1}{\left|y_{0}-x\right|}$ with $R_{0}$ given by (3.7). Let us now compute $\Phi^{\prime \prime}\left(s_{0}\right)$. We have $\Phi^{\prime}(s)=\frac{\gamma(s)-x}{|\gamma(s)-x|} \cdot \frac{1}{\rho} \gamma^{\prime}(s)$, so $\Phi^{\prime \prime}\left(s_{0}\right)=\left.\left(\mathrm{D}_{Z} \frac{Z}{|Z|}\right)\right|_{z=y_{0}-x} \gamma^{\prime}\left(s_{0}\right) \cdot \frac{\gamma^{\prime}\left(s_{0}\right)}{\rho}+\frac{y_{0}-x}{\left|y_{0}-x\right|} \cdot \frac{\gamma^{\prime \prime}\left(s_{0}\right)}{\rho}=\Psi_{1}+\Psi_{2}$. The first term is $\Psi_{1}=$ $\frac{1}{\rho\left|y_{0}-x\right|^{3}}\left|\left(y_{0}-x\right) \cdot \mathcal{R}_{-\pi / 2}^{T} \gamma^{\prime}\left(s_{0}\right)\right|^{2}$. But $\frac{y_{0}-x}{\left|y_{0}-x\right|}=-\nu_{y_{0}}=-\mathcal{R}_{-\pi / 2} \gamma^{\prime}\left(s_{0}\right)$, so $\Psi_{1}=\frac{1}{\rho\left|y_{0}-x\right|}$. By the way $\gamma^{\prime \prime}\left(s_{0}\right)=-\kappa_{0} \nu_{y_{0}}$ where $\kappa_{0}$ denotes the curvature of $\partial D$ at specular point $y_{0}$; thus $\Psi_{2}=\frac{\kappa_{0}}{\rho}$. Then for $x$ large enough, $\Psi_{1}$ is negligible compared to $\Psi_{2}$. As a result:

$$
u_{+}(x) \sim \frac{1}{\sqrt{\pi \omega \sqrt{\varepsilon_{0} \mu_{0}} \kappa_{0}}} \frac{R_{0}}{4} \frac{-1}{\left|y_{0}-x\right|} \mathrm{e}^{2 \mathrm{i} \omega \sqrt{\varepsilon_{0} \mu_{0}}\left|y_{0}-x\right|+\mathrm{i} \pi / 4} .
$$

More particularly, this finally implies the following model for the monostatic scattered intensity:

$$
I[D](x) \sim \frac{1}{4^{2} \pi \omega \sqrt{\varepsilon_{0} \mu_{0}}} \frac{1}{\left|y_{0}-x\right|^{2}} \frac{R_{0}^{2}}{\kappa_{0}}, \quad x \in \mathcal{C}(M \lambda) .
$$


To conclude this section, let us add several comments about the limits of the asymptotic-kind model that we have just derived. First, it is based on the Kirchhoff approximation. This approximation takes into account only the primary reflections on the visible part of the object. Some other contributions are neglected: the object is penetrable, so there might be also internal multiples coming back to the receiver; by the way, there may be creeping waves generated at the interface light/shadow. Concerning the visible part, calculus are approximated using a geometrical optics model followed by a stationary phase analysis. For both of these asymptotics, we need to specify what "large" means. The Rayleigh criterion commonly admitted is that a length $L$ is large if the dimensionless quantity $\frac{L}{\lambda}=\frac{k L}{2 \pi}$ is greater than $\frac{1}{4}$, where $\lambda$ is the wavelength and $k$ is the wavenumber. For the geometrical optics approximation, we thus require that $\frac{1}{\kappa \lambda} \geqslant \frac{1}{4}$; for the stationary phase method, the second derivative $\left|\Phi^{\prime \prime}\left(s_{0}\right)\right|$ cannot be too small, i.e. $|\Lambda|\left|\Phi^{\prime \prime}\left(s_{0}\right)\right|=2 k \kappa_{0} \geqslant \pi$. These two conditions can be summarized on a condition on the radius of curvature: $\frac{\lambda}{4} \leqslant \frac{1}{\kappa} \leqslant \frac{4}{\lambda}$. By the way, the stationary phase analysis should eventually take into account the contributions from other eventual critical points such as the end points of integration. Here, we have neglected them. For all these reasons, the intensity model (3.13) could be not exact, both qualitatively and quantitatively. Nonetheless it is of interest to us: it can be used as a canonic model to describe monostatic scattered intensity data; we shall just keep in mind that it is not completely exact.

\section{INVERSION METHODS}

4.1. Consequences of the asymptotic model. Before designing inversion methods to reconstruct the object, let us analyse the canonical model (3.13) that describes intensity measurements as a product of three factors. Such an analysis should give an understanding of the data as a function of the unknonwn object. This should also emphasise what kind of informations about the object is reconstructable or not, and this should lead to inversion methods.

The first factor $\frac{1}{4^{2} \pi \omega \sqrt{\varepsilon_{0} \mu_{0}}}$ depends only on the slowness of free space and the frequency wich are both assumed to be fixed. So it is a constant. The use of multi-frequency data could be eventually subject to further studies.

The second factor $\frac{1}{\left|y_{0}-x\right|^{2}}$ is a decay factor in $\frac{1}{r^{2}}$. Because of the assumption: $\left|y_{0}\right| \ll|x|=M \lambda$, we have roughly the following approximation: $\left|y_{0}-x\right|^{2} \sim(M \lambda)^{2}$. So the amplitude decay factor can be almost considered as a constant when $x$ moves on $\mathcal{C}(M \lambda)$, this constant does not depend on the object. The advantage is that it simplifies analysis, since informations are then only in the third factor.

The third factor $\frac{R_{0}^{2}}{\kappa_{0}}$ is the one which contains directly the informations about the object: it is the ratio of the (squared) reflection coefficient $R_{0}^{2}$ and the curvature $\kappa_{0}$ at specular point. The first conclusion is that we cannot hope to reconstruct both the reflection coefficient and the size of the object: multiplying $\kappa$ by a positive constant and $R_{0}$ by a square root of this constant would yield similar measurements! By the way, if $\kappa_{0}$ is supposed to be known then $R_{0}^{2}$ can be expressed as known quantities multiplied by the measurements ; next, averaging over $\theta$ estimates $R_{0}^{2}$. If on the contrary we know $R_{0}^{2}$, then the new information in the measurements is the curvature $\kappa$. Thus the inverse problem becomes: reconstructing a curve from its curvature. This will be one of the imaging method that we study below.

Unfortunately, none of these three factors depend sensitively on the position of the object; so roughly speaking, the given data do not contain the position of the object and thus it cannot be recovered! More data are needed. If we now have a closer look at the amplitude decay factor under our small scene assumption, we can see it as $\frac{1}{|\hat{y}-x|^{2}}$. The position information $\hat{y}$ is somehow hidden here. If we assume that the measurements are taken on two concentric circles (and not only one), then the data from the first circles can be mapped onto the data from the second one. The mapping is directly related to the position $\hat{y}$ due to the amplitude decay, and so this may help to recover $\hat{y}$. This is the basis of the first imaging method.

4.2. Mapping intensities from one circle to another one. In this subsection, we assume that we know the monostatic intensity $I[D]$ on two large $\operatorname{circles} \mathcal{C}\left(M_{1} \lambda\right)$ and $\mathcal{C}\left(M_{2} \lambda\right)$ with $M=M_{1}<M_{2}$. We denote $I_{1,2}$ the intensities that we measure. We try to reconstruct the position $\hat{y}$ of the object $D$ by finding a map which transforms $I_{1}$ onto $I_{2}$. 


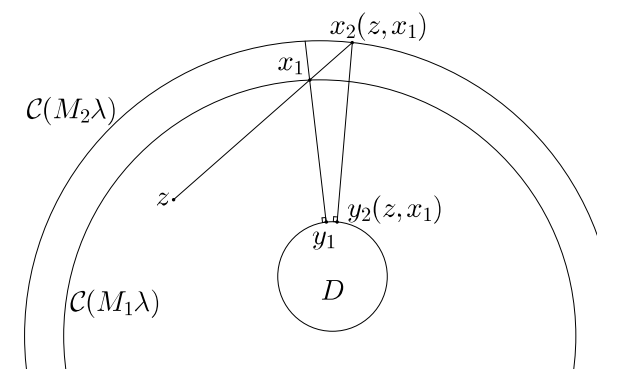

Figure 2. Configuration to map intensities: $D$ is the object, $x_{1}$ is a source/receiver on the first circle $\mathcal{C}\left(M_{1} \lambda\right)$ of measurements, $z$ is a test point, $x_{2}\left(z, x_{1}\right)$ is on the second circle $\mathcal{C}\left(M_{2} \lambda\right)$ and on the ray $\left[z, x_{1}\right), y_{1}$ and $y_{2}\left(z, x_{1}\right)$ are the associated specular points.

For a test point $z$ in the smallest disk, we "transport" $I_{1}$ along rays emanating from $z$ by applying them a correction due to the $1 / r^{2}$ decay. See Figure 2. If $x_{1}$ is a point on the first circle $\mathcal{C}\left(M_{1} \lambda\right)$, we compute the intersection of the ray $\left[z, x_{1}\right)$ with the second circle $\mathcal{C}\left(M_{2} \lambda\right): x_{2}\left(z, x_{1}\right)=z+(-z \cdot v+$ $\left.\left[\left(M_{2} \lambda\right)^{2}-|z|^{2}+(z \cdot v)^{2}\right]^{1 / 2}\right) v$, with $v=\frac{x_{1}-z}{\left|x_{1}-z\right|}$. The intensity $I_{1}\left(x_{1}\right)$ is mapped onto $\frac{\left|x_{1}-z\right|^{2}}{\left|x_{2}\left(z, x_{1}\right)-z\right|^{2}} I_{1}\left(x_{1}\right)$ and we compared it with the measurement at $x_{2}$ by computing

$$
\delta\left(z, x_{1}\right)=I_{2}\left(x_{2}\left(z, x_{1}\right)\right)-\frac{\left|x_{1}-z\right|^{2}}{\left|x_{2}\left(z, x_{1}\right)-z\right|^{2}} I_{1}\left(x_{1}\right) .
$$

We then compute the $L^{2}$ error (with respect to $x_{1}$ ) over the full circle:

$$
\|\delta(z, \cdot)\|=\left(\int\left|\delta\left(z, x_{1}\right)\right|^{2} \mathrm{~d} x_{1}\right)^{1 / 2}
$$

The idea is that the test point $z$ is close to the object $D$ when its associated mapping yields a small error $\|\delta(z, \cdot)\|$. As a result, we plot the map of

$$
H(z)=1 /\|\delta(z, \cdot)\|
$$

and we select a high level set of $H$ to get an estimate of $D$, and more particularly of its position $\hat{y}$.

Let us have a look at what happens using the asymptotic model (3.13). The model yields directly: $I_{1}\left(x_{1}\right) / I_{2}\left(x_{2}\right)=\frac{\kappa_{2}}{\kappa_{1}} \frac{\left|x_{2}-y_{2}\right|^{2}}{\left|x_{1}-y_{1}\right|^{2}}$. Here, $x_{2}=x_{2}\left(z, x_{1}\right), y_{1,2}$ is the specular point associated with $x_{1,2}$, and $\kappa_{1,2}$ is the associated curvature. If $z$ is close to $D$, the specular point $y_{2}$ is close to the specular point $y_{1}$, and so are the curvatures $\kappa_{1}$ and $\kappa_{2}$. By the way, by the small scene assumption, $\frac{\left|x_{2}-y_{2}\right|^{2}}{\left|x_{1}-y_{1}\right|^{2}} \sim \frac{\left|x_{2}-\hat{y}\right|^{2}}{\left|x_{1}-\hat{y}\right|^{2}}$ and since $z$ is close to both $y_{1}$ and $y_{2}$, we have $\frac{\left|x_{2}-\hat{y}\right|^{2}}{\left|x_{1}-\hat{y}\right|^{2}} \sim \frac{\left|x_{2}-z\right|^{2}}{\left|x_{1}-z\right|^{2}}$. Finally $I_{1}\left(x_{1}\right) / I_{2}\left(x_{2}\right) \sim \frac{\left|x_{2}-z\right|^{2}}{\left|x_{1}-z\right|^{2}}$ and so $\delta\left(z, x_{1}\right)$ should be small. On the contrary, when the test point $z$ moves away from the object $D$, the ratio $\frac{\left|x_{2}-z\right|^{2}}{\left|x_{1}-z\right|^{2}}$ moves away from $\frac{\left|x_{2}-y_{2}\right|^{2}}{\left|x_{1}-y_{1}\right|^{2}}$, or $y_{2}$ moves away from $y_{1}$ and so the curvature $\kappa_{2}$ moves away from $\kappa_{1}$; in any case there is generically no reason to have $\delta\left(z, x_{1}\right)$ small. These qualitative comments using the asymptotic model tend to confirm that $H$ is a good imaging function to find the position.

4.3. Reconstruction from curvatures. In this subsection, we come back to the original problem whose data are recorded on the unique circle $\mathcal{C}(M \lambda)$, and we suppose that we know the (squared) reflection coefficient $R_{0}^{2}$. The starting point is the asymptotic formula (3.13) wich links the intensity $I(\theta)$ measured at $x(\theta)=M \lambda \theta((\cos \theta, \sin \theta) \equiv \theta \in[0,2 \pi])$ with the curvature $\kappa_{0}(\theta)$ at the associated specular point $y_{0}(\theta): I(\theta) \sim \frac{1}{\pi \omega \sqrt{\varepsilon_{0} \mu_{0}} \kappa_{0}(\theta)} \frac{R_{0}^{2}}{4^{2}} \frac{1}{\left|y_{0}(\theta)-x(\theta)\right|^{2}}$. Using the assumption $|\gamma| \ll M \lambda$, we have the following approximations: $\left|x(\theta)-y_{0}(\theta)\right| \nu_{y_{0}}(\theta)=x(\theta)-y_{0}(\theta)=M \lambda\left(\theta-\frac{\left|y_{0}(\theta)\right|}{M \lambda}\right) \sim M \lambda \theta$. More particularly, the normal vector at specular point is assimilated to the measurement angle $\theta$ : $\nu_{y_{0}(\theta)} \sim \theta$; also we have $\left|y_{0}(\theta)-x(\theta)\right|^{2} \sim(M \lambda)^{2}$. Then the asymptotics above provides the (radius of) curvature as a function of the measurements, parametrized by the normal $\theta$ :

$$
\frac{1}{\kappa_{0}(\theta)} \sim \pi \omega \sqrt{\varepsilon_{0} \mu_{0}} M^{2} \frac{4^{2}}{R_{0}^{2}} I(\theta) .
$$


Thus, the inverse problem is equivalent to reconstructing the boundary $\partial D$ (or $\gamma$ ), from its radius of curvature $\frac{1}{\kappa_{0}(\theta)}$, parametrized by the exterior normal vector $\theta$. Here we insist on the lack of knowledge of the position of the object: the curvature contains only derivatives; so the position is missing, and it cannot be reconstructed here.

We can formulate the problem as an ODE. Indeed, by the chain rule:

$$
\frac{\mathrm{d} \gamma}{\mathrm{d} \theta}=\frac{\mathrm{d} s}{\mathrm{~d} \theta} \frac{\mathrm{d} \gamma}{\mathrm{d} s}=\frac{1}{\kappa_{0}(\theta)}(-\sin \theta, \cos \theta)
$$

and so we would like to find a solution to the following ODE:

$$
\frac{\mathrm{d} \gamma}{\mathrm{d} \theta}=\frac{1}{\kappa_{0}(\theta)}(-\sin \theta, \cos \theta)=: f(\theta), \quad \gamma(\theta) \text { is } 2 \pi \text {-periodic. }
$$

Before we can hope to find such a periodic function, the right member $f(\theta)$ must be of zero mean. Here the curvature $\kappa_{0}(\theta)$ is only roughly known, so unfortunately this may be unsatisfied. By the way, even if we authorize non-periodic solutions, the constant of integration cannot be determine by this method because we cannot determine the position of the object. Because of these facts of non-existence or non-uniqueness, a regularization is needed.

Let us now write the discrete version of the ODE problem that we have to solve. The measurement angle $\theta$ lives in the discrete set: $\left\{\theta_{i}:=i h, 0 \leqslant i \leqslant n\right\}$ with $h=\frac{2 \pi}{n+1}$. These angles correspond also to the normal vectors at specular points. The boundary $\gamma$ evaluated on those specular points is the $(n+1) \times 2$ unknown $Y=\left(\gamma\left(\theta_{i}\right)\right)_{i}$. The discretized right member is the $(n+1) \times 2$ matrix $F=\left(f\left(\theta_{i}\right)\right)_{i}$. And the discrete operator associated with the (periodic) ODE is a circulant matrix of size $(n+1) \times(n+1)$, corresponding to a finite differences discretization:

$$
K=\frac{1}{2 h}\left(\begin{array}{cccc}
0 & 1 & & -1 \\
-1 & 0 & \ddots & \\
& \ddots & \ddots & 1 \\
1 & & -1 & 0
\end{array}\right)
$$

With these notations, the discrete problem is the linear system: find the $(n+1) \times 2$ matrix $Y$ such that

$$
K Y=F .
$$

If $n+1$ is odd, then the constant vector $(1, \ldots, 1)$ spans the kernel of $K$. Else, the kernel of $K$ is spanned by the family $\{(1, \ldots, 1),(1,0,1,0, \ldots, 1,0)\}$. In both cases, the matrix is not of full rank, and then there cannot be a unique solution!

We then propose a Tikhonov-Phillips regularization. We have to assume that we have already determined some estimate $Y_{0}$ of $Y$ by another method ; $Y_{0}$ could eventually be a constant representing the position, eventually determined by the mapping method presented above. We use this a priori information in an optimization problem: find the $(n+1) \times 2$ matrix $Y_{\sigma}$ wich minimizes the cost function:

$$
J_{\sigma}(Y):=\frac{1}{2}\|K Y-F\|^{2}+\frac{\sigma}{2}\left\|Y-Y_{0}\right\|^{2} .
$$

Here, $\sigma>0$ is a fixed parameter, and for a $(n+1) \times 2$ matrix $X=\left(X_{1}, X_{2}\right)$, we have defined $\|X\|^{2}:=\left|X_{1}\right|^{2}+\left|X_{2}\right|^{2}$. The first term of this functionnal tends to impose $Y$ to reproduce the observed data, whereas the second term tends to force $Y$ to be near $Y_{0}$. The parameter $\sigma$ can be chosen to make some compromise between the two terms. A point $Y$ is a minimizer over the full space of such a convex functionnal if, and only if, it satisfies the Euler equation $J_{\sigma}^{\prime}(Y)=0$, wich reduces here to the linear system:

$$
\left(K^{*} K+\sigma \mathrm{I}\right) Y=K^{*} F+\sigma Y_{0},
$$

where $K^{*}$ is the adjoint matrix of $K$. Since the matrix $K^{*} K+\sigma \mathrm{I}$ is positive definite, then the linear system is invertible. The matrix $K^{*} K+\sigma \mathrm{I}$ is a sparse symetric circulant matrix, whose first line is: $\left(\frac{1}{2 h^{2}}+\sigma, 0, \frac{-1}{4 h^{2}}, 0, \ldots, 0, \frac{-1}{4 h^{2}}, 0\right)$. Thus the system can be efficiently solved, eventually by using the fast fourier transform. Its solution $Y_{\sigma}$ is finally the unique solution to the minimization problem and can be used to estimate the boundary $\partial D$. 


\section{Numerical Results}

5.1. Boundary element method versus geometrical optics. We check numerically the relevance (or the limits) of the asymptotic model (3.12). So we compute the scattered wave $u_{+}$on the circle $\mathcal{C}(M \lambda)$ using this model and another reference method. The reference result $u_{\mathrm{MoM}}$ is computed by the boundary element method, and is parametrized by the angular position $\theta$ of the point $x(\theta)$ on the circle. Concerning the asymptotic result $u_{\text {Curvature, }}$, we compute it by using finite differences to evaluate the curvature $\kappa=\left|\mathcal{R}_{-\pi / 2} \dot{y} \cdot \ddot{y}\right| /|\dot{y}|^{3}$, where $y(t), 0 \leqslant t \leqslant 2 \pi$ is a parametrization of $\partial D$, and the dot is the derivative with respect to $t$. $\partial D$ is first discretized by $Y=\left(y\left(t_{i}\right)\right)_{0 \leqslant i \leqslant N}$, with $t_{i}=i h, h=\frac{2 \pi}{N+1}$. Then let $K$ be the $(N+1) \times(N+1)$ derivation matrix of the form (4.1), and let $\Delta$ be the $(N+1) \times(N+1)$ matrix of the second derivative operator: $\Delta=\frac{1}{h^{2}}\left(\begin{array}{cccc}-2 & 1 & & 1 \\ 1 & -2 & \ddots & \\ & \ddots & \ddots & 1 \\ 1 & & 1 & -2\end{array}\right)$. Then, $\left(\dot{y}\left(t_{i}\right)\right)_{i}=K Y+O\left(h^{2}\right)$, and $\left(\ddot{y}\left(t_{i}\right)\right)_{i}=\Delta Y+O\left(h^{2}\right)$. This immediately gives $\left(\kappa\left(t_{i}\right)\right)_{i}$, and then we get the asymptotics $u_{\text {Curvature, }}$ parametrized by $t_{i}$. (NB : we deduce the normal vector at $y\left(t_{i}\right)$ from $\dot{y}\left(t_{i}\right)$; so we get the angle $\theta$ whose associated specular point is $y\left(t_{i}\right)$, and thus $u_{\text {Curvature }}$ can be parametrized by $\theta$ too.)

We start by observing the effect of the reflection coefficient $R_{0}$, wich is closely related to the permeability contrast $\frac{\mu_{*}}{\mu_{0}}$ and the slowness contrast $\frac{k_{*}}{k_{0}}=\frac{\sqrt{\varepsilon_{*} \mu_{*}}}{\sqrt{\varepsilon_{0} \mu_{0}}}$. We choose the parameters as follows. The wavelength is $\lambda=0.5$. The permeability of free space is $\mu_{0}=1$. The contrast of slowness is $\frac{k_{*}}{k_{0}}=2$. The object is an ellipsoid whose diameter is the large axis $a_{1}=10 \lambda$. The small axis is of size $a_{2}=0.7 a_{1}$. The large axis makes an angle of 1 with the $x$ axis, and the center of the ellipsoid is $(10 \lambda,-3 \lambda)$. The object is discretized with about 12 points per wavelength (and at least 50 points). The scattered wave is computed on the circle $\mathcal{C}(M \lambda)$ whose radius divided by the wavelength is $M=1000$. This circle of measurements is uniformly discretized with 360 points. As a quantitative criterion to compare the methods, we compute the relative error between the reference result $u_{\mathrm{MoM}}$ and the asymptotic result $u_{\text {Curvature }}:\left|u_{\mathrm{MoM}}-u_{\text {Curvature }}\right| /\left|u_{\mathrm{MoM}}\right|$ (ratio of $L^{2}$ norms on $\mathcal{C}(M \lambda)$ ). Here, we try several reflection coefficients by changing the contrast $\frac{\mu_{*}}{\mu_{0}}$. We get approximately the following results:

\begin{tabular}{c|cccc}
$\frac{\mu_{*}}{\mu_{0}}$ & 1.5 & 0.8 & 0.2 & 0.02 \\
$R_{0}$ & -0.14 & -0.43 & -0.82 & -0.98 \\
\hline$\left|u_{\mathrm{MoM}}-u_{\text {Curvature }}\right| /\left|u_{\mathrm{MoM}}\right|$ & 0.96 & 0.74 & 0.43 & 0.15
\end{tabular}

We have also plotted $u_{\mathrm{MoM}}$ and $u_{\text {Curvature }}$ on the Figure 3 , for the cases $\frac{\mu_{*}}{\mu_{0}}=1.5$ and $\frac{\mu_{*}}{\mu_{0}}=0.02$. These different tests tend to reveal that contributions neglected by the Kirchhoff approximation can seriously affect the scattered wave, for both the amplitude and the phase. We also see that the results are better for a reflection coefficient close to minus one. For such a coefficient, the situation is indeed closer to the perfectly reflecting one where the Kirchhoff approximation is more reasonable.

We now test the effect of the asymptotics requirement (including $\frac{\lambda}{4} \leqslant \frac{1}{\kappa} \leqslant \frac{4}{\lambda}$ ) by testing several ratios size of the object over the wavelength. We only indicate the parameters wich are different from those in the tests above. We set the permeability contrast: $\frac{\mu_{*}}{\mu_{0}}=0.02$, and so the reflection coefficient is $R_{0} \approx-0.98$. The used wavelength is $\lambda=0.75$. The ratio $\frac{a_{1}}{\lambda}$ takes now several successive values. The relative error of the asymptotic $u_{\text {Curvature }}$ is:

\begin{tabular}{c|cccc}
$\frac{a_{1}}{\lambda}$ & 1 & 5 & 10 & 15 \\
\hline$\left|u_{\mathrm{MoM}}-u_{\text {Curvature }}\right| /\left|u_{\mathrm{MoM}}\right|$ & 1.1 & 0.47 & 0.15 & 0.24
\end{tabular}

We see again some limits of the asymptotic model: the error increases when the curvature becomes too small or too large.

Unfortunately, the global conclusion is that the canonical model (3.12) gives results whose quality is variable. The optimistic point of view is that results are quite good in certain regimes of parameters; this allows to justify the following imaging methods at least for those classes of parameters. 

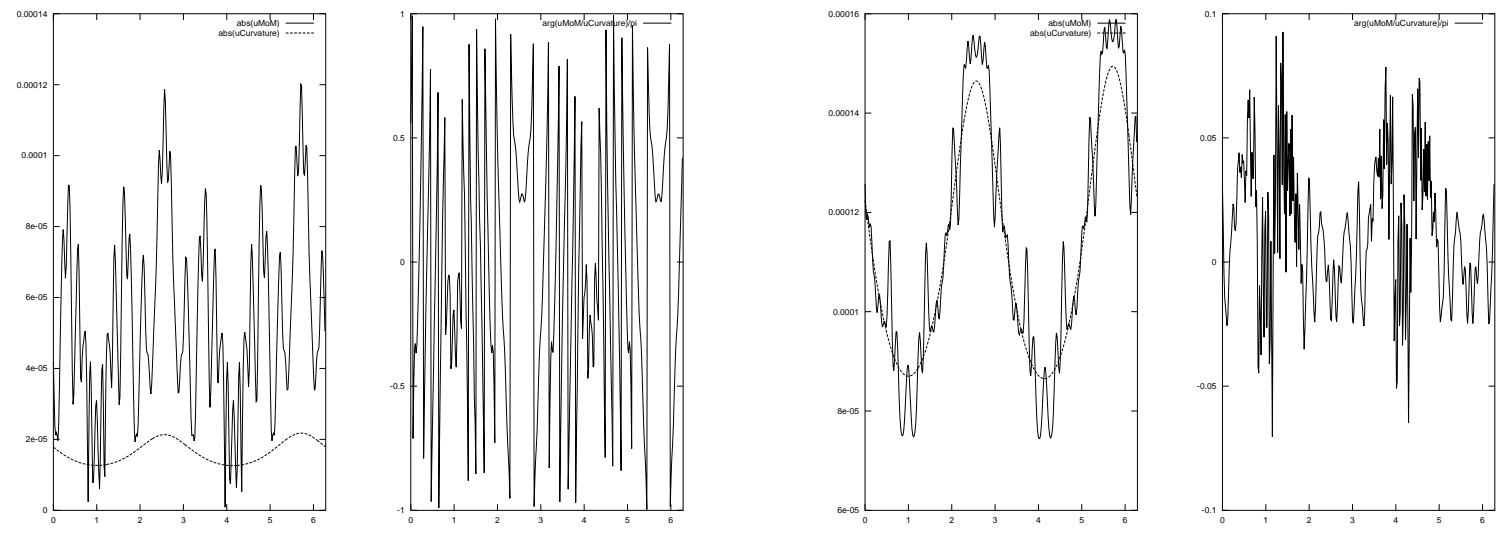

Figure 3. Comparison of the monostatic wave reflected by an ellipsoid, computed with the boundary element method $\left(u_{\mathrm{MoM}}\right)$ and the Kirchhoff approximation ( $\left.u_{\text {Curvature }}\right)$. The reflection coefficient is $R_{0} \approx-0.14$ on the left and $R_{0} \approx-0.98$ on the right. For both cases, we plot the modules on the left and we plot the difference of the arguments divided by $\pi$ and on the right. For $R_{0} \approx-0.14$, the two results are quite different. For $R_{0} \approx-0.98$, the results are quite close.
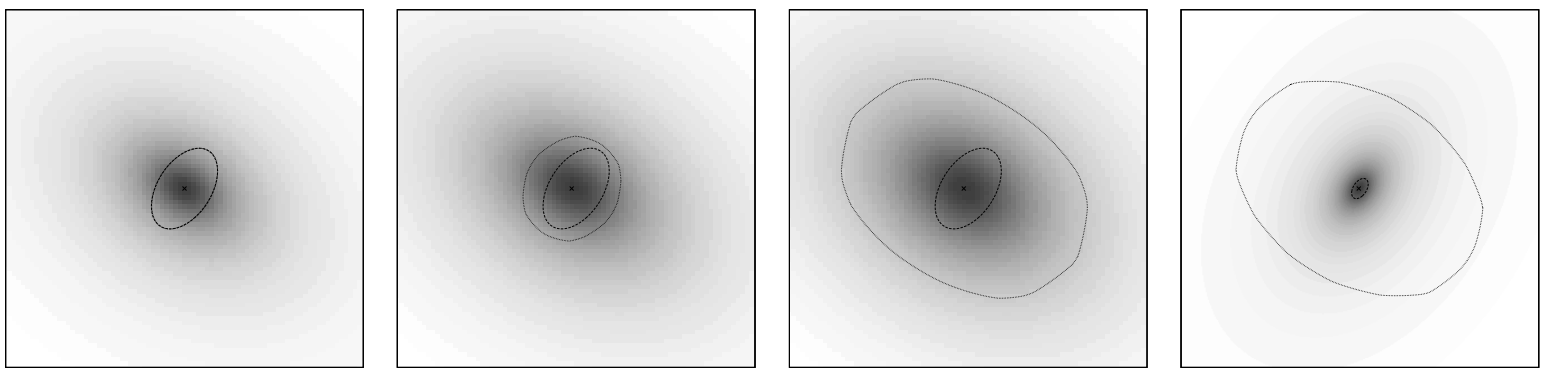

FIGURE 4. Imaging for different reflection coefficients: $R_{0} \approx-0.98,-0.82,-0.43,-0.14$.

5.2. Inversion. We now test numerically the imaging methods. Monostatic scattered intensities $I_{1,2}$ on the circles $\mathcal{C}\left(M_{1,2} \lambda\right)$ are simulated using the boundary element method, the object $D$ being discretized with about 12 points per wavelength $\lambda$. We first guess the position of the object using the mapping method. We plot the imaging function $H$ on a grid whose step is quarter of a wavelength: $\lambda / 4$ (on both axis). We use a grayscale; the darker is the gray, the larger is $H$. We mark with a cross the highest value of $H$. The corresponding position is used as a guess $Y_{0}$ of the position of the object. Then we use the curvature inversion method, based on this guess and on data $I_{1}$ from the first circle. The regularization parameter $\sigma$ is set to 0.01 . The resulting reconstruction is finally plotted on the same picture than $H$; and so is the true boundary $\partial D$. We explicit below different sets of parameters. Comments about the results will be done later in the text, method by method.

The following parameters will be always the same. The permeability of free space is $\mu_{0}=1$, the slowness contrast is $\frac{k_{*}}{k_{0}}=2$. The radius of the first circle of measurements is $M_{1} \lambda$ with $M_{1}=1000$. The two circles of measurements are uniformly discretized with 360 points. When the object is an ellipsoid, the ratio of the axes is $\frac{a_{2}}{a_{1}}=0.6$, and the large axis makes an angle of 1 with the $x$ axis.

We first test the methods for different reflection coefficients $R_{0}$, since it is a critical parameter for the asymptotic forward modelling. The contrast $\frac{\mu_{*}}{\mu_{0}}$ takes the successive values $0.02,0.2,0.8,1.5$ and so the reflection coefficient $R_{0}$ takes the values $-0.98,-0.82,-0.43,-0.14$. The other parameters are the following. The wavelength is $\lambda=0.5$. The object is an ellipsoid whose diameter is the large axis $a_{1}=5 \lambda$. Its center is $(10 \lambda,-3 \lambda)$. The radius of the second circle of measurements is $M_{2} \lambda=1.1 M_{1} \lambda$. The results of the imaging methods are represented on Figure 4 .

Then, we try ellipsoids whose center is $(10 \lambda,-3 \lambda)$ and whose size is variable: the ratio large axis over the wavelength takes the successice values: $\frac{a_{1}}{\lambda}=0.5,2,8,16$. By the way, the wavelength is $\lambda=0.75$. The permeability contrast is $\frac{\mu_{*}}{\mu_{0}}=0.2$; so the reflection coefficient $R_{0}$ is about -0.82 . 

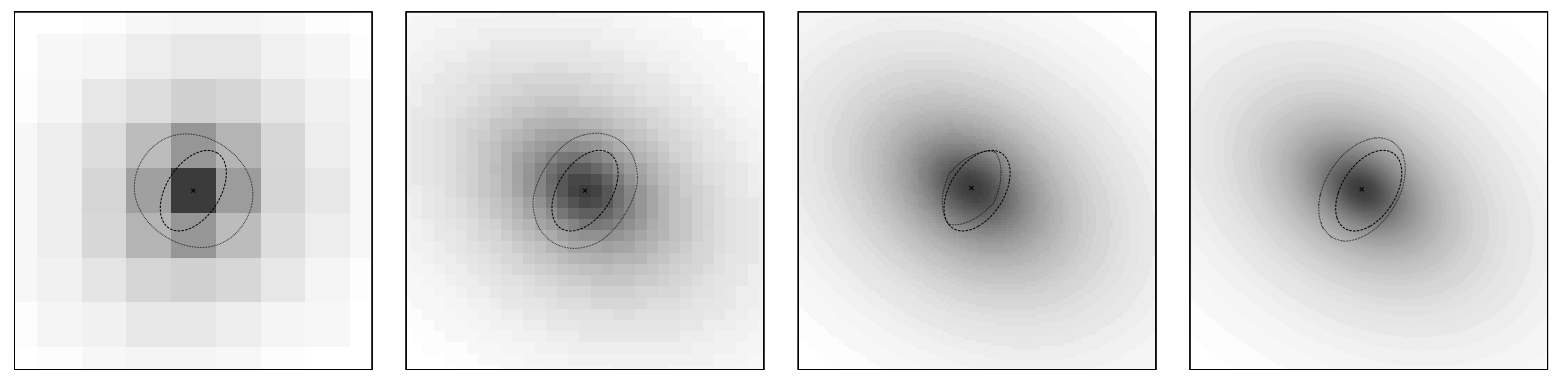

Figure 5. Imaging of an ellipsoid whose size $a_{1}$ is given by $\frac{a_{1}}{\lambda}=0.5,2,8,16$.

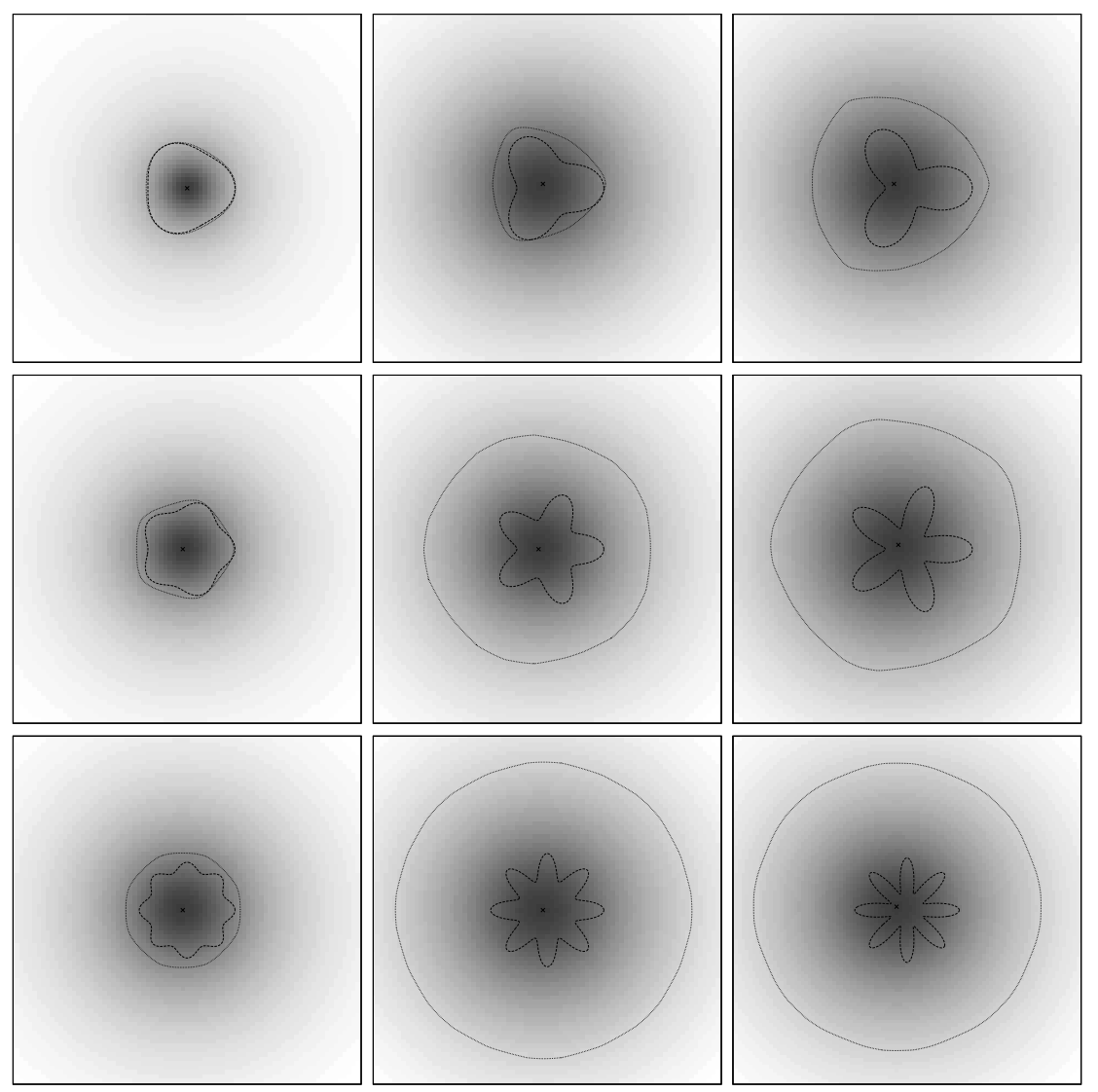

Figure 6. Imaging of a star whose number of branchs is $f=3,5,8$ (from top to bottom) and whose branch amplitude is $\alpha=0.1,0.3,0.5$ (from left to right).

The ratio of the radius of the circles of measurements is $\frac{M_{2}}{M_{1}}=1.1$. The imaging results are plotted on Figure 5.

Although the methods were designed for convex objects, we also observe numerically what happens for star-shaped objects, whose boundary is of the form: $\gamma(t)=(10 \lambda,-3 \lambda)+2.5 \lambda(1+$ $\alpha \cos (f t))(\cos t, \sin t)$. The number of branchs is $f=3,5,8$ and the amplitude of a branch is $\alpha=0.1,0.3,0.5$. The center of such a star is $(10 \lambda,-3 \lambda)$ and its diameter is about $5 \lambda$. The other parameters are the following. The wavelength is $\lambda=0.5$. The contrast $\frac{\mu_{*}}{\mu_{0}}$ is 0.02 and so the reflection coefficient $R_{0}$ is -0.98 . The ratio of the radius of the circles of measurements is $\frac{M_{2}}{M_{1}}=1.1$. The imaging results are plotted on Figure 6.

Mapping from the first circle to the second one. For all the cases, the first method provides a map whose largest values are located near the inclusion. By the way, the maximum of this map is reached near the center of the object and so its location $Y_{0}$ is a good guess of position.

We add some quality control of the mapping on one illustrative example. Let us recall that $Y_{0}$ is by definition the point $z$ such that the mapping of $I_{1}$, i.e. $\frac{\left|x_{1}-z\right|^{2}}{\left|x_{2}\left(z, x_{1}\right)-z\right|^{2}} I_{1}\left(x_{1}\right)$, is the closest from $I_{2}$ (evaluated on $\left.x_{2}\left(z, x_{1}\right)\right)$. To control the quality of this mapping, we compute the mapping of $I_{1}$ for 

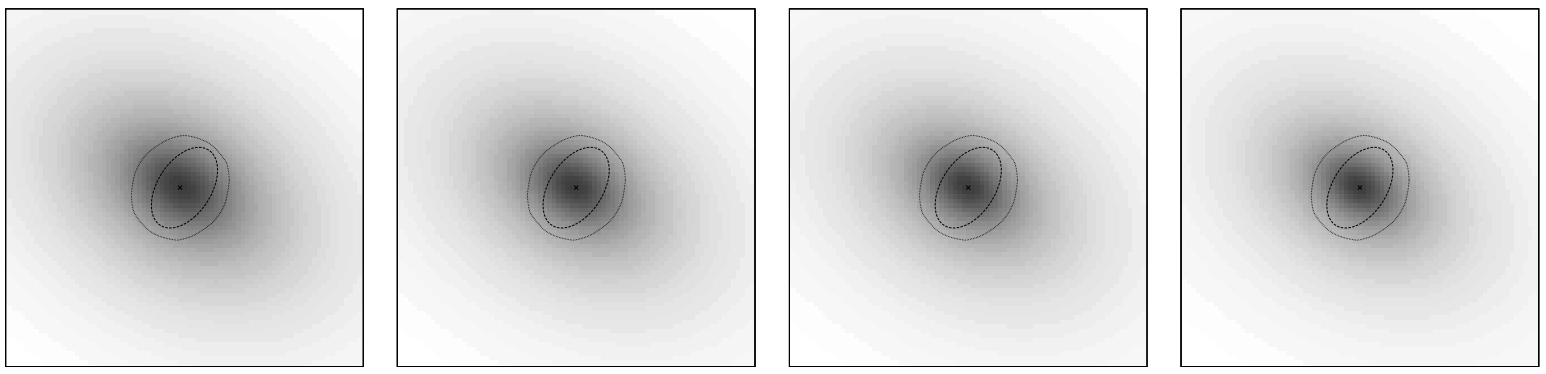

FiguRE 7. Imaging with different ratio of radius of the circles of measurements: $\frac{M_{2}}{M_{1}}=1.05,2,3,10$

$z=Y_{0}$ and we compare it with $I_{2}$ by computing the relative error $\left\|\delta\left(Y_{0}, x_{1}\right)\right\| /\left\|I_{2}\left(x_{2}\left(Y_{0}, x_{1}\right)\right)\right\|$. For the third example of Figure 4, we get $0.11 \%$ wich is very small, and which means that the mapping is very good for $z=Y_{0}$.

We add another test, about the radius of the second circle of measurements to check that the method is not sensitive to it. More precisely, the ratio of the radius $\frac{M_{2}}{M_{1}}$ takes here the successive values: $1.05,2,3,10$. The other parameters are the following. The wavelength is $\lambda=0.5$. The contrast $\frac{\mu_{*}}{\mu_{0}}$ is 0.2 and so the reflection coefficient $R_{0}$ is -0.82 . The object is an ellipsoid whose center is $(10 \lambda,-3 \lambda)$ and whose large axis is $a_{1}=5 \lambda$. The results of the Figure 7 shows that the method is not sensitive at all to the ratio of radius. The only effect of increasing it is to very slightly concentrate the highest values of the imaging function near the center of the object.

The conclusion is that the method finds the position and that it is very robust.

Inversion from curvature. First, even if the estimate of the curvature from intensities data is very noisy, we always reconstruct a curve which is smooth. We also observe that the reconstruction is always convex. This is due to the method itself which defines the curvature as a positive number. The reconstruction is also well located, due to the (good) guess of the position $Y_{0}$ wich is used as an a priori. We can claim that the regularization parameter $\sigma$ has been well chosen.

For the ellipsoids of Figure 5, all the results are quite good. (For the first image, the shape is not so well reconstructed, but the error is under the resolution limit $\lambda / 4$.) The method was indeed designed for such convex objects with a quite good reflection coefficient. By the way, the reflection coefficient is critical: on Figure 4, the reconstructed curve is perfect for $R_{0}=-0.98$, but deteriorates when $R_{0}$ approaches 0 . This is due to the Kirchhoff forward model wich deteriorates. And to finish with, when the non-convexity increases, the forward model deteriorates again. That is why for the stars on Figure 6, the reconstruction is very good for the first image but deteriorates more and more when the number of branchs or/and the branch amplitude increase.

\section{REFERENCES}

[1] H. Ammari. An Introduction to Mathematics of Emerging Biomedical Imaging. Springer, 2008.

[2] H. Ammari, P. Garapon, F. Jouve, H. Kang, M. Lim, and S. Yu. A new optimal control approach for the reconstruction of extended inclusions. SIAM Journal on Control and Optimization, 51:1372-1394, 2013.

[3] H. Ammari, J. Garnier, W. Jing, H. Kang, M. Lim, K. Sølna, and H. Wang. Mathematical and Statistical Methods for Multistatic Imaging. Lecture Notes in Mathematics, Springer, submitted.

[4] H. Ammari, J. Garnier, V. Jugnon, and H. Kang. Stability and resolution analysis for a topological derivative based imaging functional. SIAM Journal on Control and Optimization, 50:48-76, 2012.

[5] H. Ammari, J. Garnier, H. Kang, M. Lim, and K. Sølna. Multistatic imaging of extended targets. SIAM Journal on Imaging Sciences, 5:564-600, 2012.

[6] J.-B. Bellet and G. Berginc. Imagerie laser. C. R. Acad. Sci. Paris, 2011.

[7] N. Bleistein, J. Cohen, and J. Stockwell Jr. Mathematics of Multidimensional Seismic Imaging, Migration, and Inversion, volume 13. Springer, 2000.

[8] N. Bleistein and R. A. Handelsman. Asymptotic Expansions of Integrals. Dover, 1986.

[9] A. Chai, M. Moscoso, and G. Papanicolaou. Array imaging using intensity-only measurements. Inverse Problems, 27(1):015005, 2011.

[10] J. R. Fienup et al. Phase retrieval algorithms: a comparison. Applied optics, 21(15):2758-2769, 1982.

[11] F. Friedlander and J. B. Keller. Asymptotic expansions of solutions of $\left(\nabla^{2}+k^{2}\right) u=0$. Communications on Pure and Applied Mathematics, 8(3):387-394, 1954.

[12] G. Gbur and E. Wolf. Diffraction tomography without phase information. Optics letters, 27(21):1890-1892, 2002. 
[13] A. Majda. High frequency asymptotics for the scattering matrix and the inverse problem of acoustical scattering. Communications on Pure and Applied Mathematics, 29(3):261-291, 1976.

[14] L. Pan, Y. Zhong, X. Chen, and S. P. Yeo. Subspace-based optimization method for inverse scattering problems utilizing phaseless data. Geoscience and Remote Sensing, IEEE Transactions on, 49(3):981-987, 2011.

[15] M. R. Weiss. Inverse scattering in the geometric-optics limit. JOSA, 58(11):1524-1527, 1968.

Institut Elie Cartan de Lorraine (site de Metz), Université de Lorraine, UMR CNRS 7502, 57045 Metz Cedex 1 (France)

E-mail address: jean-baptiste.bellet@univ-lorraine.fr

Thales Optronique, 2, Avenue Gay Lussac CS 90502, 78995 Élancourt Cedex (France)

E-mail address: gerard.berginc@fr.thalesgroup.com 